\title{
A patellar tendon length conservation method: Biplanar retrotubercle open-wedge proximal tibial osteotomy
}

\author{
Is Ismail Turkmen, ${ }^{1}$ (i) Irfan Esenkaya ${ }^{2}$ \\ ${ }^{1}$ Department of Orthopaedics and Traumatology, Health Sciences University Umraniye Training and Research Hospital, Istanbul, Turkey \\ ${ }^{2}$ Department of Orthopaedics and Traumatology, Istnbul Medeniyet University Faculty of Medicine, Istanbul, Turkey
}

\begin{abstract}
OBJECTIVE: The early-period results of our technique for performing a medial biplanar retrotubercle open-wedge proximal tibial osteotomy for the surgical treatment of varus gonarthrosis were evaluated and compared with those reported in the literature.

METHODS: The clinical and radiological results of a medial biplanar retrotubercle open-wedge proximal tibial osteotomy performed on 23 knees in 22 patients with medial gonarthrosis with varus alignment were analyzed.

RESULTS: Twenty patients were female and 2 were male. At the time of surgery, the mean age was 56.22 years (44-66 years), the mean body mass index was $31.95 \mathrm{~kg} / \mathrm{m}^{2}\left(23.4-44.9 \mathrm{~kg} / \mathrm{m}^{2}\right)$, and the mean Hospital for Special Surgery (HSS) score was 68.7 (48-83). The mean preoperative femorotibial anatomical axis angle was $186.39^{\circ}\left(173-194^{\circ}\right)$, and the mean Insall-Salvati index value was 1.04 (0.94-1.171). The mean length of follow-up was 30.19 months (6-42 months). At the last follow-up examination, the mean HSS score was 86.48 (74-100), the mean femorotibial anatomical axis angle was $175^{\circ}$ $\left(168-171^{\circ}\right)$, and the mean Insall-Salvati index value was $1.06(0.857-1.32)$. Comparison of the final follow-up values with the preoperative values demonstrated significant improvement in the HSS score and femorotibial anatomical axis angle, but no significant difference in the Insall-Salvati index value.

CONCLUSION: The results of this study indicated that frontal and sagittal plane deformities in patients with varus gonarthrosis can be treated with biplanar retrotubercle open-wedge proximal tibial osteotomy with good clinical results that achieve patellar tendon length stability and avoid patellofemoral problems.

Keywords: Biplanar; open-wedge osteotomy; patellar tendon; retrotubercle.

Cite this article as: Turkmen I, Esenkaya I. A patellar tendon length conservation method: Biplanar retrotubercle open-wedge proximal tibial osteotomy. North Clin Istanb 2018;5(3):246-253.
\end{abstract}

Ceveral alternative treatments are available for medial gonarthrosis. They include proximal tibial osteotomy (PTO), which is a "biological treatment" option often used for relatively young patients. PTO techniques have also been modified many times. Recently, medial openwedge osteotomies have been preferred because they produce very good clinical results [1]. However, patients may have patellofemoral problems when the osteotomy is performed only in a single plane [2]. The purpose of this study was to demonstrate that good clinical results were obtained when treating a frontal plane deformity with our technique of medial biplanar retrotubercle open-wedge PTO, without changing the length of the patellar tendon in the sagittal plane.

Received: November 04, 2017 Accepted: February 27, 2018 Online: August 31, 2018

Correspondence: Dr. Ismail TURKMEN. Saglik Bilimleri Universitesi, Umraniye Egitim ve Arastirma Hastanesi, Ortopedi ve Travmatoloji Klinigi, Istanbul, Turkey. 


\section{MATERIALS AND METHODS}

The ethics committee of Istanbul Medeniyet University, Goztepe Training and Research Hospital approved a review and analysis of patients diagnosed with medial gonarthrosis. Informed consent was obtained from each patient. The results of medial biplanar retrotubercle openwedge PTO performed on patients with medial gonarthrosis and varus alignment who were treated in the clinic were examined. A single surgeon, who is the developer of the technique and one of the authors, performed the operation on all of the patients. The technique is a modification of the method defined by Gaasbeek et al. [2,3].

The study inclusion criteria were knee pain, arthrosis in the medial compartment of the knee, varus alignment, and nonresponse to conservative treatment. Exclusion criteria were grade 3 or 4 chondropathy in the lateral compartment according to the Outerbridge classification as determined by arthroscopy or magnetic resonance imaging (MRI) [4], knee flexion contracture $>15^{\circ}$, knee flexion $<90^{\circ}$, femoral varus deformity, or serious arterial or venous insufficiency.

Initially, 30 patients with medial gonarthrosis and varus malalignment were included. Six patients were excluded after the first step of the surgery due to the observation of grade 4 chondropathy on the lateral side of the knee during arthroscopy or they had not undergone an osteotomy. Two patients were also lost to follow-up. Thus, the study was completed with the data of 23 knees of 22 patients.

The clinical examination included details obtained from the patients' medical records and history: the nature of each patient's complaint, date of onset, history of prior medication use, previous treatment, presence of other diseases, and the use of cigarettes. Each patient's height, weight, and body mass index (BMI) was also recorded. The Hospital for Special Surgery Knee Rating Sheet (HSS score) was used to assess knee function [5].

The radiological assessment consisted of anteroposterior (AP) and lateral radiographs of both knees taken while the patient was in the standing and $30^{\circ}$ flexion positions. Orthoroentgenography was performed in both lower extremities in the routine manner. If not performed previously, MRI and computed tomography imaging were completed before surgery as usual. Before surgery, the femoral and tibial anatomical axes, the angle between the femoral condyle and the tibial plateau (FCTPa), the angle between the tibial plateau and the

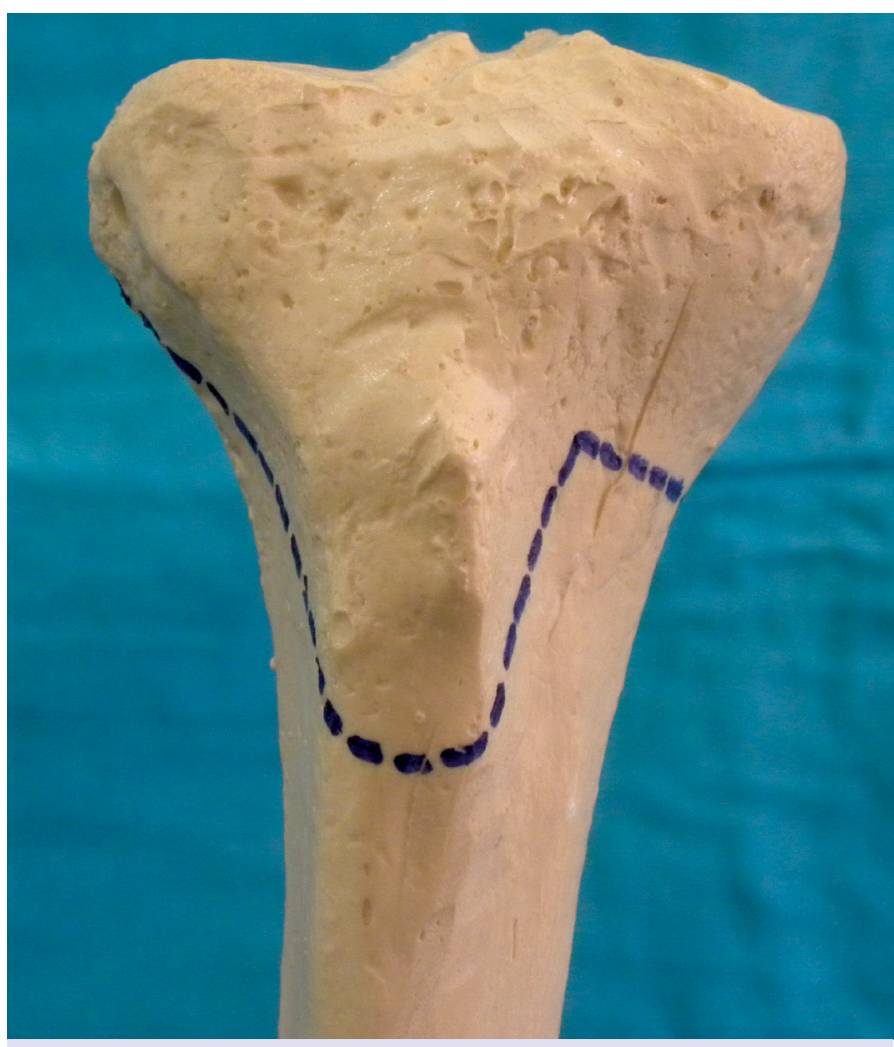

FIGURE1. The appearance of the osteotomy line on the bone model - anterior side.

tibial axis (TPTAa), the tibial slope, and Insall-Salvati index parameters were measured.

All of the patients underwent arthroscopy before the osteotomy. Microfracture surgery and chondroplasty were performed on patients with grade 4 chondropathy in the medial compartment. Once the decision was made to perform an osteotomy, an approximately $8-\mathrm{cm}$ anterior longitudinal skin incision was made immediately below the area between the tuberositas tibia and the lower pole of the patella (this incision shape does not prevent possible future total knee prosthesis placement). The tibial periosteum and pes anserinus were elevated in an inverse " $\mathrm{L}$ " shape. The superficial fibers of the medial collateral ligament were cut. In the first scope control, the first Kirschner wire (K-wire) was passed from the medial region of the proximal tibial metaphysis $(4 \mathrm{~cm}$ below the joint line) toward the lateral cortex such that it stayed 1 to $1.5 \mathrm{~cm}$ distal to the joint and about $1 \mathrm{~cm}$ medial to the lateral cortex. If it was passed at a right angle in the AP or lateral plane, the wire was shortened and additional $\mathrm{K}$ wires were passed accordingly. The $\mathrm{K}$-wires were placed so that they would stay in the proximal tibial tubercle and the osteotomy would be biplanar. The anterior wire 


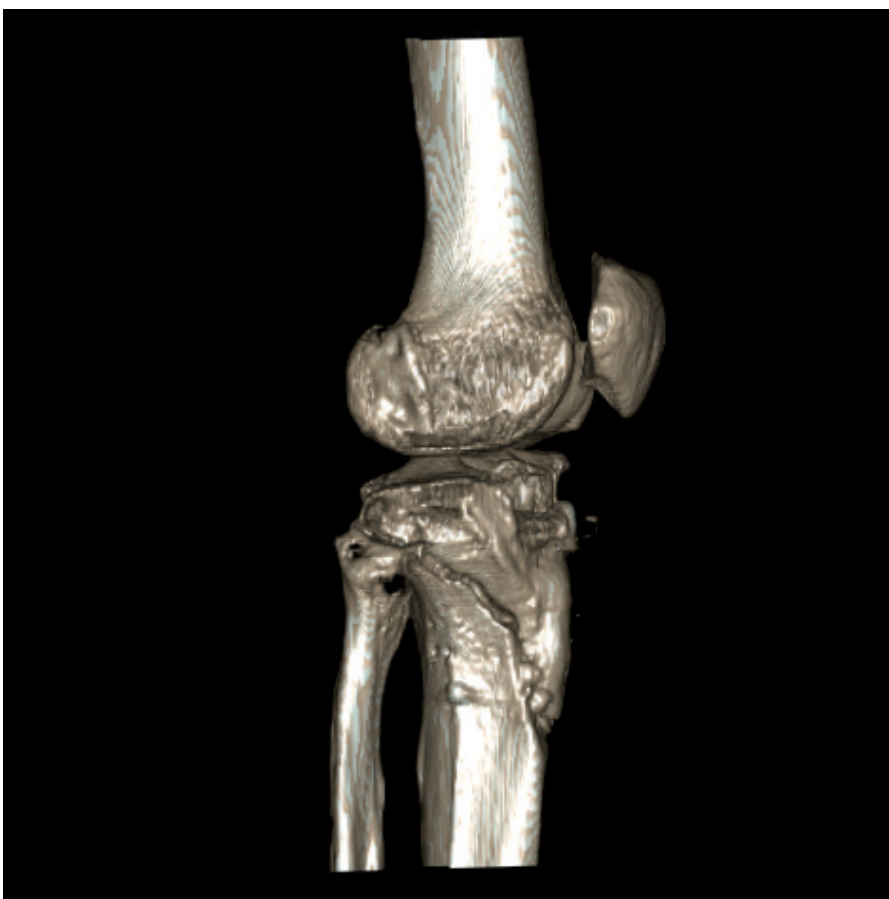

FIGURE 2. The appearance of the osteotomy line on 3-dimensional computed tomography - lateral side.

was left in the anterior third of the tibial metaphysis. The incisional peak behind the tubercle should be at least 2.5 $\mathrm{cm}$ and the angle between the horizontal and vertical osteotomy lines should be 110 to $120^{\circ}$ (Figs. 1, 2) [3, 6]. If the angle is smaller, the tibial tubercle may break; if the angle is larger, the application of a double plate is more difficult, due to the decreased bone angle [3]. The vertical osteotomy line was osteotomized with the thinnest osteotome. The posterior tibial cortex was dissected with a specially designed blunt-ended osteotome to protect posterior neurovascular structures. The horizontal osteotomy line was opened using a triple osteotome technique or a specially designed distractor [7].

The amount of correction was planned preoperatively, and distraction at the osteotomy site was performed according to the preoperative plan. The wedge-shaped gap remaining after fixation was filled with a bicortical autograft taken from the iliac crest. Fixation was enabled through specially designed wedge plates $[3,6,8-10]$. Tibial tubercules could not be fixed. The medialized distal end of the tibial tubercule was trimmed (Fig. 4).

\section{RESULTS}

Twenty patients in this study group were female and 2

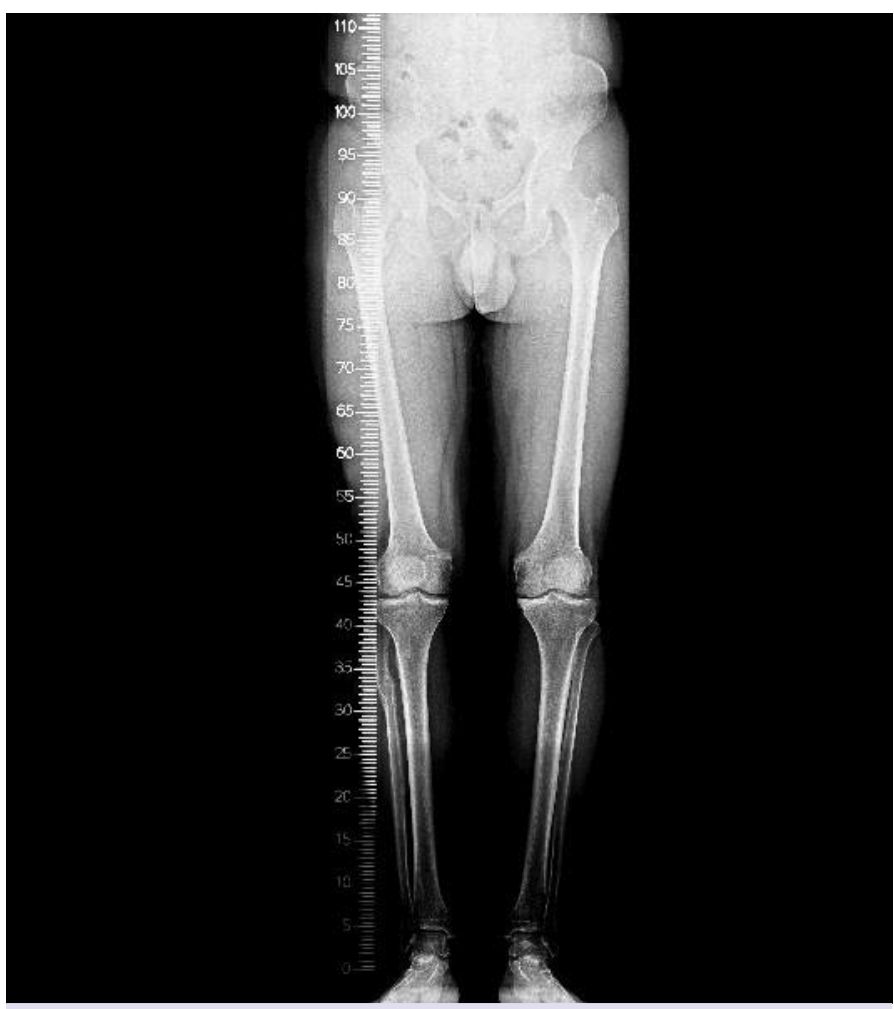

FIGURE 3. A 58-year-old male patient who was operated on from the left side due to bilateral genu varum.

\begin{tabular}{lcccccccc}
\hline & HSS & TFAAa & FCTPaO & TPTAa & SLOPE & $\begin{array}{r}\text { INSALL PLATE } \\
(\mathrm{mm})\end{array}$ \\
Preoperative & 78 & 186 & 7 & 97 & 13 & 1.00 & $9+11$ \\
Postoperative & 91 & 173 & 6 & 89 & 11 & 1.05 & \\
\hline
\end{tabular}

Preoperative anteroposterior graphy in a standing position.

FCTPa: Angle between the femoral condyle and tibial plateau; HSS: Hospital for Special Surgery; Insall: Insall-Salvati index; TFAAa: Tibiofemoral anatomic axis angle; TPTAa: Angle between the tibial plateau and the tibial axis; Slope: Tibial slope.

were male. The right knee of 12 patients, the left knee of 9 patients, and both knees of 1 patient were operated on. The arthroscopic findings of the patients were evaluated and are presented in Table 1.

The postoperative HSS scores were significantly higher than the preoperative scores $(p<0.05)$. The postoperative mean flexion and extension values showed no significant change from the baseline figures (both $\mathrm{p}>0.05$; Table 2 ). The mean preoperative TPTAa was 97.17 $\pm 3.85^{\circ}$ (median: $98^{\circ}$ ) and the mean TPTAa at the final follow up was $88.09 \pm 5.23^{\circ}$ (median: $88^{\circ}$ ). The mean change was $-9.09 \pm 5.28^{\circ}\left(-17^{\circ}\right.$ to $7^{\circ}$; median: $-10^{\circ}$; Table 3). No meaningful correlation was found between the change in HSS score (postoperative vs. pre- 

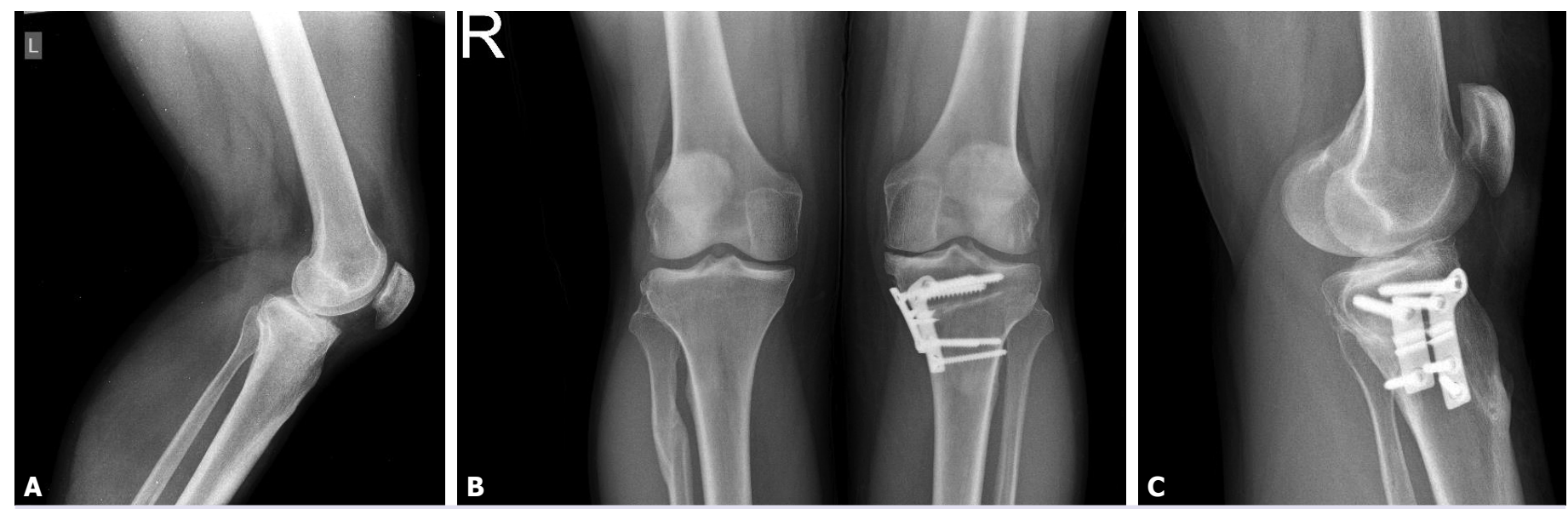

FIGURE 4. (A) Left knee lateral graphy. (B) Postoperative month 9 anteroposterior standing graphy. (C) Postoperative month 9 left knee lateral graphy.

TABLE 1. Arthroscopic findings in 23 knees of 22 patients

\begin{tabular}{|c|c|c|c|c|c|}
\hline & $\mathrm{n}$ & $\%$ & & $\mathrm{n}$ & $\%$ \\
\hline \multicolumn{6}{|l|}{ Chondropathy in FMC } \\
\hline $\mathrm{G} 3 / 3-4$ & 7 & 30.4 & ACL rupture & 1 & 4.3 \\
\hline G 4 & 16 & 69.6 & PCL rupture & 0 & 0.0 \\
\hline \multicolumn{6}{|l|}{ Chondropathy in TMP } \\
\hline G 3 & 8 & 34.8 & Plica & 4 & 17.4 \\
\hline G 4 & 15 & 65.2 & Ligamentum mucosum & 0 & 0.0 \\
\hline Medial meniscus lesion & 14 & 60.9 & Microfracture & 21 & 91.3 \\
\hline Lateral meniscus lesion & 5 & 21.7 & & & \\
\hline Retropatellar chondropathy & 5 & 21.7 & & & \\
\hline Trochlear chondropathy & 4 & 17.4 & & & \\
\hline
\end{tabular}

ACL: Anterior cruciate ligament; FMC: Femoral medial condyle; PCL: Posterior cruciate ligament; TMP: Tibial medial plateau.

operative) and the change in flexion, extension, TFAAa, FCTPa, TPTAa, tibial slope, or Insall-Salvati index value (all $\mathrm{p}>0.05$; Table 4).

Complications: The following complications occurred in this sample of 23 knees in 22 patients: non-fatal pulmonary embolism $(n=1)$, deep vein thrombus 1 year after surgery $(n=1)$, temporary sensory nerve injury $(n=2$; lateral femoral cutaneous nerve hypoesthesia in 1 patient and hypoesthesia in the infrapatellar branch of the saphenous nerve in 1 patient), rhabdomyolysis $(n=1)$, and loss (recurrence) at a recoverable level $(n=1)$. No surface or deep infection, lateral tibial cortex or plateau fracture, tibial tubercle fracture, or plate or screw breakdown was observed.

\section{DISCUSSION}

Patients who undergo PTO may have deformities in not just the frontal plane, but also in the sagittal plane [11]. After medial open-wedge PTO, the tibial slope tends to increase [12]. Giffin et al. [13] reported a $4.4^{\circ}$ increase in slope postoperatively compared with the preoperative value, Naudie et al. [14] reported a $7.7^{\circ}$ increase, and Bombaci et al. [15] reported a mean increase of $3.6^{\circ}$. Insufficiency of the posterior cortex and insufficient loosening of the posterior soft tissues play a role in this change [12]. The increase in tibial slope increases anterior subluxation of the tibia. Whereas this increase is positive in cases of a posterior cruciate ligament failure, a 
TABLE 2. Comparison of the preoperative and postoperative Hospital for Special Surgery scores and the flexion and extension assessments

\begin{tabular}{|c|c|c|c|c|c|}
\hline & Mean \pm SD & Median & Min.-Max. & $\mathrm{p}$ & \\
\hline \multicolumn{6}{|l|}{ HSS score } \\
\hline Preoperative & $68.70 \pm 11.68$ & 70 & $48-83$ & & \\
\hline Postoperative & $86.48 \pm 9.05$ & 88 & $66-100$ & 0.000 & $\mathrm{E}$ \\
\hline Amount of change & $17.78 \pm 10.23$ & 17 & $-4-39$ & & \\
\hline \multicolumn{6}{|l|}{ Flexion } \\
\hline Preoperative & $110.65 \pm 15.32$ & 120 & $70-130$ & & \\
\hline Postoperative & $114.13 \pm 11.55$ & 120 & $85-130$ & 0.275 & w \\
\hline Amount of change & $3.48 \pm 12.65$ & 0 & $-15-30$ & & \\
\hline \multicolumn{6}{|l|}{ Extension } \\
\hline Preoperative & $-0.43 \pm 2.09$ & 0 & $-10-0$ & & \\
\hline Postoperative & $-0.43 \pm 1.44$ & 0 & $-5-0$ & 1.000 & w \\
\hline Amount of change & $0.00 \pm 1.51$ & 0 & $-5-5$ & & \\
\hline
\end{tabular}

SD: Standard deviation; Min.: Minimum; Max.: Maximum; HSS: Hospital for special surgery.

TABLE 3. The postoperative and preoperative TFAAa, FCTPa, TPTAa, slope, and Insall-Salvati index change

\begin{tabular}{|c|c|c|c|c|c|}
\hline \multicolumn{6}{|l|}{ TFAAa } \\
\hline Postoperative & $175.00 \pm 5.54$ & 175 & 168-191 & \multirow[t]{2}{*}{0.000} & \multirow[t]{2}{*}{$\mathrm{E}$} \\
\hline Amount of change & $-11.39 \pm 5.06$ & -10 & $-23--3$ & & \\
\hline Postoperative & $4.09 \pm 1.73$ & 5 & $1-7$ & \multirow[t]{2}{*}{0.000} & \multirow[t]{2}{*}{$\mathrm{E}$} \\
\hline Amount of change & $-2.13 \pm 1.55$ & -2 & $-6-0$ & & \\
\hline \multicolumn{6}{|l|}{ TPTAa } \\
\hline Preoperative & $97.17 \pm 3.85$ & 98 & $91-105$ & 0.000 & $\mathrm{E}$ \\
\hline Preoperative & $12.96 \pm 4.38$ & 12.0 & $7.0-24.0$ & \multirow{3}{*}{0.560} & \multirow{3}{*}{$\mathrm{E}$} \\
\hline Postoperative & $13.70 \pm 5.71$ & 13.0 & $7.0-36.0$ & & \\
\hline Amount of change & $0.74 \pm 5.99$ & 0.0 & $-5.0-25.0$ & & \\
\hline \multicolumn{6}{|l|}{ Insall-Salvati index } \\
\hline Preoperative & $1.04 \pm 0.08$ & 1.1 & $0.9-1.2$ & \multirow{3}{*}{0.057} & \multirow{3}{*}{$E$} \\
\hline Postoperative & $1.06 \pm 0.13$ & 1.1 & $0.9-1.3$ & & \\
\hline Amount of change & $0.03 \pm 0.07$ & 0.0 & $-0.1-0.2$ & & \\
\hline
\end{tabular}

SD: Standard deviation; Min.: Minimum; Max.: Maximum; HSS: Hospital for special surgery; E: Matched sample t-test; FCTPa: Angle between the femoral condyle and the tibial plateau; TFAAa: Tibiofemoral anatomic axis angle; TPTAa: Angle between the tibial plateau and the tibial axis. 
TABLE 4. The relationship between radiological and clinical findings in the preoperative and postoperative periods

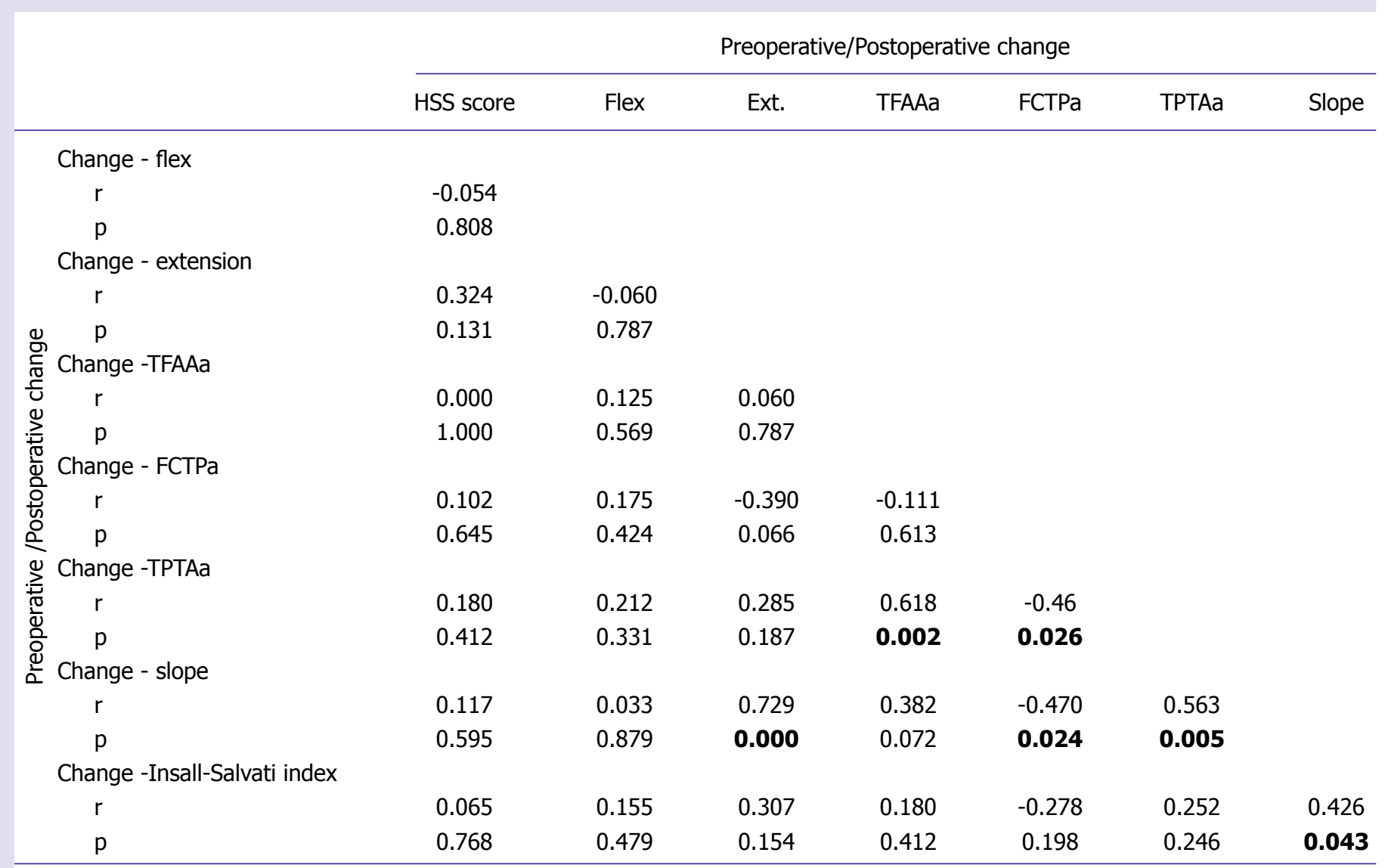

Pearson/Spearman correlation; FCTPa: Angle between the femoral condyle and tibial plateau; HSS: Hospital for Special Surgery; TFAAa: Tibiofemoral anatomic axis angle; TPTAa: Angle between the tibial plateau and the tibial axis; Slope: Tibial slope.

decrease in tibial slope is positive in cases of anterior cruciate ligament failure and extension limitation [11, 12]. In 2006, Esenkaya and Elmali [16] reported that they used a technique involving placement of a plate with a small $(2-2.5 \mathrm{~mm})$ wedge in front of the osteotomy line to prevent an increase in the tibial slope. We also used this technique to avoid changing the tibial slope. There is no need to change the slope in patients who have full knee extension. If there is a desire to increase or decrease the tibial slope, a double plate technique should be used. Otherwise, a single rectangular plate can be used. In our study, the difference between preoperative and postoperative tibial slope was not significant $(p=0.560)$. One patient had a partial anterior cruciate ligament rupture, but no instability; thus, we did not change the tibial slope in this patient.

Medial open-wedge osteotomy is used for acute fixation [1-3, 6, 10, 12, 14-17] and gradual fixation [18].
Fixation plates are used for acute fixation $[2,3,6,10,12$, $14,17]$ and external fixation systems are used for gradual fixation [18]. Acute fixation is divided into monoplanar $[10,15-17]$ and biplanar $[2,3,6,11]$ types. Biplanar osteotomy can preserve the proximal $[2,3,6]$ or distal [11] tibial tubercle. The use of retrotubercle osteotomy began because this technique does not change the location of the patella or the Q angle [2,3]. Murphy [19] preferred this type of osteotomy for wedge closure. Then, Gaasbeek et al. [2] used this technique in an open-wedge osteotomy. In a medial open-wedge osteotomy performed from the proximal tibial tubercle, the tubercle is reduced by half of the deformity degree [20]. Thus, narrowing of the patellar tendon and patella infera can be observed [21]. This increases retropatellar pressure and causes patellofemoral arthrosis $[3,22]$. Patella infera can create technical problems for total knee prosthesis surgery [2]. To avoid patellar tendon length shortening, Hernigou et 
al. [1] oriented the graft with the greatest wedge height to the far back of the osteotomy, and used grafts with shorter $(2 \mathrm{~mm})$ wedges in the middle and at the far front. However, because this technique does not involve the use of a fixation plate after the osteotomy, tibial slope change is inevitable. In patients with patellofemoral arthrosis or the need for $<15^{\circ}$ fixation, an osteotomy that preserve the location of the proximal patellar tendon attachment is preferred [3]. In our study, we used a technique developed by Gaasbeek et al. [2] that was modified by the senior surgeon/author, with wedge plates designed by the senior surgeon/author (patent number: TR2002 02021Y). The Insall-Salvati index was used to evaluate the change in patellar tendon size. The mean preoperative and postoperative Insall-Salvati index value, as calculated from radiographs, was $1.04 \pm 0.08^{\circ}$ (median: $1.1^{\circ}$ ) and $1.06 \pm 0.13^{\circ}$ (median: $1.1^{\circ}$ ), respectively. The difference was not significant $(p=0.057)$. One patient had recoverable loss, with a change in the sagittal plane and an increased Insall-Salvati index score. The inclusion of data from this patient in the statistical analysis did not change the mean statistical result.

In our study, 1 (4.5\%) patient had a non-fatal pulmonary embolism, 1 (4.5\%) patient developed deep vein thrombus 1 year after surgery, $2(9.1 \%)$ patients had temporary sensory nerve injury (lateral femoral cutaneous nerve hypoesthesia in 1 patient and hypoesthesia in the infrapatellar branch of the saphenous nerve in the other patient), 1 (4.5\%) patient had rhabdomyolysis, and $1(4.5 \%)$ patient had loss (recurrence) at a recoverable level; in total, this amounted to $6(27.2 \%)$ complications in 22 patients. The pulmonary embolism caused no serious clinical issue, and no thrombus was present in the leg veins at the time of diagnosis. When the embolism occurred, the patient received $0.4 \mathrm{~mL}$ enoxaparin sodium daily. The deep vein thrombus occurring 1 year after surgery was treated symptomatically. Nerve injury was determined in the nerves feeding the related sensory areas and was resolved without treatment. One patient was found to have rhabdomyolysis, which began with oliguria and was detected with renal function tests on postoperative day 2 . This patient was treated with early dialysis. The clinical view had improved markedly at postoperative month 7 , but the femoral muscles were atrophied compared with the other side. We associated the formation of rhabdomyolysis with the use of a tourniquet [23]. We observed a loss in the degree of improvement in 1 patient. Loading was not performed with this patient, who had a high BMI, but no implant insufficiency was observed. Retrospective examination revealed that a 30 cc allograft was used in this patient. We found no fracture or implant insufficiency in any patient. Although the plates we used were not locking plates, no screw loss occurred. The plates had a thin profile, and none of our patients developed skin problems.

Our study has some limitations. First, it was retrospective and lacked a control group. Although a followup period of 6 months is sufficient to assess early clinical results, a longer period would make the study more valuable. Although our study included more patients than some other studies, the sample size was still small.

In the surgical treatment of varus gonarthrosis, PTO may be considered before prosthesis options. PTOs can be applied with different shapes, grafts, and implants. Our study findings indicate that frontal and sagittal plane deformities in patients with varus gonarthrosis can be treated with biplanar retrotubercle open-wedge PTO, which can achieve patellar tendon length stability, avoid patellofemoral problems, and obtain good clinical results.

The English in this document has been checked by at least two professional editors, both native speakers of English. For a certificate, please see:

http://www.textcheck.com/certificate/irLeN5

Conflict of Interest: All authors declare that there is no conflict of interest.

Financial Disclosure: The authors declared that this study has received no financial support.

Authorship Contributions: Concept - I.E., Design - I.T.; Supervision - I.E.; Materials - I.T.; Data collection \&/or processing - I.T.; Analysis and/or interpretation - I.E.; Writing - I.T.; Critical review - I.E.

\section{REFERENCES}

1. Hernigou P, Medevielle D, Debeyre J, Goutallier D. Proximal tibial osteotomy for osteoarthritis with varus deformity. A ten to thirteen-year follow-up study. J Bone Joint Surg Am 1987;69:332-54. [CrossRef]

2. Gaasbeek RD, Sonneveld H, van Heerwaarden RJ, Jacobs $\mathrm{WCH}$, Wymenga AB. Distal tuberosity osteotomy in open wedge high tibial osteotomy can prevent patella infera: a new technique. Knee 2004;11:6:457-61. [CrossRef]

3. Esenkaya I, Unay K. Proximal medial tibial biplanar retrotubercle open wedge osteotomy in medial knee arthrosis. Knee 2012;19:416-21.

4. Outerbridge RE. The etiology of chondromalacia patellae. J Bone Joint Surg Br 1961;43:752-7. [CrossRef]

5. Insall JN, Dorr LD, Scott RD, Scott WN. Rationale of the Knee Society clinical rating system. Clin Orthop Relat Res 1989;248:13-4.

6. Esenkaya I, Unay K, Turkmen I. Retro Tubercle Open Wedge Osteotomy in Medial Gonarthrosis. Turkiye Klinikleri J Orthop \& Traumatol-Special Topics 2013;6:79-85. 
7. Esenkaya I. A new distractor with angle-scale for proximal tibia medial opening wedge osteotomy. Knee Surg Sports Traumatol Arthrosc 2006;14:443-6. [CrossRef]

8. Esenkaya I, Misirlioglu M, Kelestemur MH, Elmali N, Fadillioglu E. Biomechanical evaluation of different fixation plates in medial opening upper tibial osteotomy. Knee 2007;14:46-50. [CrossRef]

9. Yardimeden A, Kelestemur MH, Esenkaya I. Biomechanical comparison of the wedge supported plates at PTO. Archives of Material Science Engineering 2007;28:495-8.

10. Staubli AE, Simoni CD, Babst R, Lobenhoffer P. TomoFix: a new LCPconcept for open wedge osteotomy of the medial proximal tibia--early results in 92 cases. Injury 2003;34:55-62. [CrossRef]

11. Lobenhoffer P, De Simoni C, Staubli AE. Open-wedge high-tibial osteotomy with rigid plate fixation. Tech Knee Surg 2002;1:93-105.

12. Marti CB, Gautier E, Wachtl SW, Jacob RP. Accuracy of frontal and sagittal plane correction in open-wedge high tibial osteotomy. Arthroscopy 2004;20:366-72. [CrossRef]

13. Giffin JR, Vogrin TM, Zantop T, Woo SL, Harner CD. Effects of increasing tibial slope on the biomechanics of the knee. Am J Sports Med 2004;32:376-82. [CrossRef]

14. Naudie DDR, Amendola A, Fowler PJ. Opening wedge high tibial osteotomy for symptomatic hyperextension-varus thrust. Am J Sports Med 2004;32:60-70. [CrossRef]

15. Bombaci H, Canbora K, Onur G, Görgeç M. The effect of open wedge osteotomy on the posterior tibial slope. Acta Orthop Traumatol Turc
2005;39:404-10.

16. Esenkaya I, Elmali N. Proximal tibia medial open-wedge osteotomy using plates with wedges: early results in 58 cases. Knee Surg Sports Traumatol Arthrosc 2006;14:995-61. [CrossRef]

17. Koshino T, Murase T, Saito T. Medial opening-wedge high tibial osteotomy with use of porous hydroxyapatite to treat medial compartment osteoarthritis of the knee. J Bone Joint Surg Am 2003;85:78-85.

18. Catagni MA, Guerreschi F, Ahmad TS, Cattaneo R. Treatment of genu varum in medial compartment osteoarthritis of the knee using the Ilizarov method. Orthop Clin North Am 1994;25:509-14.

19. Murphy SB. Tibial osteotomy for genu varum. Indications, preoperative planning, and technique. Orthop Clin North Am 1994;25:477-82.

20. Franco V, Cerullo G, Cipolla M, Gianni E, Puddu G. Open wedge high tibial osteotomy. Tech Knee Surg. 2002;1:43-53. [CrossRef]

21. Elmali N, Esenkaya I, Can M, Karakaplan M. Monoplanar versus biplanar medial open-wedge proximal tibial osteotomy for varus gonarthrosis: a comparison of clinical and radiological outcomes. Knee Surg Sports Traumatol Arthrosc 2013;21:2689-95. [CrossRef]

22. Esenkaya I, Elmali N, Misırlığlu M, Ertem K, Atasever A. The Medial Opening Wedge Osteotomy in Which The Tibial Tubercle Remains Attached to the Proximal Fragment: An Anatomic Study [Article in Turkish]. İnönü Üniversitesi Tip Fakültesi Dergisi 2005;12:153-7.

23. Türkmen İ, Esenkaya İ, Unay K, Akçal MA. Rhabdomyolysis after tourniquet use in proximal tibial osteotomy: a case report and review of the literature. Acta Orthop Traumatol Turc 2015;49:338-41. [CrossRef] 